K. NOMURA

KODAI MATH. J.

1 (1978), 354-361

\title{
INNER SUBGROUPS OF FINITE GROUPS
}

\author{
By KAZUMASA NOMURA
}

\section{$\S 1$. Introduction}

Let $G$ be a finite group, and $H$ be a subgroup of $G$. We call $H$ an inner subgroup of $G$, when every automorphism of $H$ can be extended to an inner automorphism of $G$. The purpose of this paper is to prove the following theorems.

THEOREM 1. Let $G$ be a finite group. If every subgroup of $G$ is an inner subgroup of $G$, then $G$ is isomorphic to the symmetric groups $S_{1}, S_{2}$ or $S_{3}$.

THEOREM 2. Let $G$ be a finte solvable group. If every abelian subgroup of $G$ is an inner subgroup of $G$, then $G$ is isomorphic to the symmetric groups $S_{1} S_{2}$, $S_{3}$ or the quaternion group $Q_{8}$.

Theorem 3. Let $G$ be a finite group, and $S$ be a 2-Sylow subgroup of $G$. Suppose every abelian subgroup of $G$ is an inner subgroup of $G$, and every abelian normal subgroup of $S$ is cyclic. Then $G$ is isomorphic to the symmetric groups $S_{1}, S_{2}, S_{3}$ or the quaternion group $Q_{8}$.

It is interesting to classify all finite groups whose every abelian subgroup is an inner subgroup. It seems that $S_{1}, S_{2}, S_{3}$ and $Q_{8}$ are the only examples of such groups. A more interesting problem is to classify all finite groups whose every cyclic subgroup is an inner subgroup. This condition is equivalent to that the character values of the groups are all rational. But this problem seems to be very difficult.

Theorem 1 is a corollary of Theorem 2 and the following proposition.

Proposition 1. If every Sylow subgroup of a finte group $G$ is inner subgroup of $G$, then $G$ is metacyclic.

This proposition will be proved by using a theorem of Gaschütz on automorphisms of p-groups. In the proof of Theorem 2, we shall use Theorem 3.

In the following, every notation is standard and can be found in [1]. Only elementary results are assumed, in paticular Sylow's theorem, properties of $p$ groups and automorphisms of abelian groups.

All groups will be finite.

Received July 13, 1977. 


\section{§2. Preliminaries}

Definition. A subgroup $H$ of a group $G$ is an inner subgroup of $G$ (or inner in $G)$ iff $N_{G}(H) / C_{G}(H)=\operatorname{Aut}(H)$.

Definition. A group $G$ is an $A I$-group iff every abelian subgroup of $G$ is inner in $G$.

In this section we prove some lemmas about $A I$-groups.

LEMMA 1. Let $G$ be an AI-group, and $P$ be a p-Sylow subgroup for a prime divisor $p$ of $|G|$. Then $|Z(P)|=p$.

Proof. By way of contradiction, assume $|Z(P)|>p$. Then $|\operatorname{Aut}(Z(P))|$ is divided by $p$. Let $\sigma$ be an automorphism of $Z(P)$ of order $p$. Since $Z(P)$ is an inner subgroup of $G$, there exists $x \in N_{G}(Z(P))$, such that $x$ induces $\sigma$ on $Z(P)$. We may assume $x$ is a $p$-element. Let $T$ be a $p$-Sylow subgroup of $N_{G}(Z(P))$ that contains $x$. There exists $g \in N_{G}(Z(P))$ such that $P^{g}=T$ by Sylow's theorem. Then $Z(P)=Z(P)^{g}=Z\left(P^{g}\right)=Z(T)$. Since $x \in T, x$ centralizes $Z(P)=Z(T)$. This contradicts the fact that $x$ induces an automorphism of order $p$ on $Z(P)$.

Q.E. D.

LEMMA 2. Let $G$ be an AI-group, and $p$ be a prime divisor of $|G|$, then the set of all elements of order $p$ of $G$ is a conjugate class of $G$.

Proof. Let $P$ be a $p$-Sylow subgroup of $G$. By Lemma $1, Z(P)=\langle a\rangle$ and $|\langle a\rangle|=p$. Since $\operatorname{Aut}(\langle a\rangle)$ acts on $\langle a\rangle$ transitively and $\langle a\rangle$ is an inner subgroup of $G$, all elements of $\langle a\rangle^{\#}$ are conjugate in $G$. Let $b \in P-Z(P)$ be an element of order $p$. If such $b$ does not exist, then we conclude the proof by Sylow's theorem. Hence we may assume such $b$ exists. $\langle a, b\rangle$ is an elementary abelian, $p$-group, and $\operatorname{Aut}(\langle a, b\rangle)$ acts on $\langle a, b\rangle^{\#}$ transitively. Since $\langle a, b\rangle$ is an inner subgroup of $G, a$ and $b$ are conjugate in $G$. Hence all elements of order $p$ in $P$ are conjugate in $G$. By Sylow's theorem, all elements of order $p$ in $G$ are conjugate.

Q.E.D.

LEMMA 3. Let $P$ be a p-Sylow subgroup of a group $G$ for a prime divisor of $|G|$. Let $M$ be a maximal abelian normal subgroup of $P$. Then we have $C_{G}(M)=M \times T$ for some $p^{\prime}$-group $T$. If further $G$ is an AI-group, $T$ is also an AI-group.

Proof. We first show that $M$ is a $p$-Sylow subgroup of $C_{G}(M)$. Let $Q$ be a p-Sylow subgroup of $C_{G}(M)$ such that $Q \supseteqq M$. Since $P, Q \leqq N_{G}(M)$, we have $Q^{g} \cong P$ for some $g \in N_{G}(M)$. Since $C_{G}(M)<N_{G}(M), Q^{g} \leqq C_{G}(M)^{g}=C_{G}(M)$. Hence we have $Q^{g} \subseteq C_{P}(M)$. Since $C_{P}(M)=M$ by [2] Satz (III, 12.1), we have $Q^{g} \subseteq M$ and $Q=M$. This means $M$ is a $p$-Sylow subgroup of $C_{G}(M)$. By Burnside's theorem, there exists a normal complement $T$ of $M$ in $C_{G}(M)$. It is clear that $C_{G}(M)=M \times T$ and $T$ is a $p^{\prime}$-group. 
Next we suppose $G$ is an $A I$-group, and show that $T$ is also an $A I$-group. Take any abelian subgroup $K$ of $T$ and any automorphism $\sigma$ of $T$. $H=K M$ $=K \times M$ is an abelian subgroup of $G$. We define an automorphism $\tilde{\sigma}$ of $H$ as following :

$$
\tilde{\sigma}= \begin{cases}\sigma & \text { on } K, \\ \text { identity } & \text { on } M .\end{cases}
$$

Since $H$ is an inner subgroup of $G$, we can found $x \in N_{G}(H)$ that induces $\tilde{\sigma}$ on $H$. Since $\tilde{\sigma}$ acts on $M$ trivially, we have $x \in C_{G}(M)$. Set $x=y z, y \in T$ and $z \in M$. Since $z$ acts on $K$ trivially, $y$ induces $\sigma$ on $K$. We have shown that any automorphism $\sigma$ of $T$ is induced from an element $y$ of $T$. Hence $T$ is an $A I$-group.

Q.E.D.

LEMMA 4. Every non-trivial AI-group has an even order.

Proof. If $G$ has an element $x$ of odd order, there exists $\sigma \in \operatorname{Aut}(\langle x\rangle), x^{\sigma}=x^{-1}$. Hence $\operatorname{Aut}(\langle x\rangle)$ is of even order. Since $\langle x\rangle$ is an inner subgroup of $G, G$ is also of even order.

Q. E. D.

Lemma 5. Let $G$ be an AI-group, $P$ be a 2-Sylow subgroup of $G$ and $M$ be a maximal abelian normal subgroup of $P$. Then $C_{G}(M)=M$.

Proof. We may assume $G \neq 1$. By Lemma 4, 2 is a divisor of $|G|$. By Lemma 3, we have $C_{G}(M)=M \times T$ and $T$ is of odd order. If $T \neq 1$, then $T$ is of even order by Lemma 4 , a contradiction. Hence $T=1$.

Q.E. D.

\section{§3. Proof of Proposition 1}

Let $G$ be a group whose every Sylow subgroup is inner in $G$. We shall see that every $p$-Sylow subgroup of $G$ has order $p$ for any prime divisor of $|G|$. Then every Sylow subgroup of $G$ is cyclic, hence $G$ is metacyclic.

Let $p$ be a prime divisor of $|G|$, and $P$ be a $p$-Sylow subgroup of $G$. Assume $|P|>p$. By Gaschütz' theorem ([2] Satz III, 19.1), the order of the outer automorphism group $\operatorname{Aut}(P) / \operatorname{Inn}(P)$ is divided by $p$. Hence there exists a $p$-element $\sigma \in \operatorname{Aut}(P)-\operatorname{Inn}(P)$. By assumption, $P$ is an inner subgroup of $G$. Hence we can find $x \in N_{G}(P)$ that induces $\sigma$ on $P$ by conjugation. We may assume $x$ is a $p$ element. Then the subgroup $\langle x\rangle P$ of $G$ is a $p$-group, and we have $\langle x\rangle P \supseteqq P$. Since $P$ is a $p$-Sylow subgroup, we have $\langle x\rangle P=P$ and $x \in P$. It follows that $\sigma \in \operatorname{Inn}(P)$, this contradicts the choice of $\sigma$.

Q.E.D.

Let $G$ be a group and let every subgroup of $G$ be inner. By Proposition 1, $G$ is metacyclic and hence solvable. Hence we can apply Theorem 2 to conclude that $G$ is isomorphic to $S_{1}, S_{2}, S_{3}$ or $Q_{8}$. Set $Q_{8}=\{ \pm 1, \pm i, \pm j, \pm k\}$. There exists an automorphism of $Q_{8}$ that removes $i$ to $j$, but this automorphism is not inner. In this way, we get Theorem 1 from Proposition 1 and Theorem 2. 


\section{§4. Proof of Theorem 3 .}

In this section, we assume $G$ is a non-trivial $A I$-group, and $S$ is a 2-Sylow subgroup of $G$. By Lemma $4, S$ is not trivial. We divide the proof into several steps.

(1) If $|S|=2$, then $G=S_{2}$ or $S_{3}$.

Proof. We assume $G>S$. Set $S=\langle\tau\rangle, \tau$ is an involution. There exists a normal complement $L$ of $S$ in $G$ by Burnside's theorem. Since $C_{G}(S)=S$ by Lemma $5, \tau$ acts on $L$ as a fixed-point-free automorphism of order 2. Hence $L$ is abelian ([1] Th. 10.1.4). Since $\left|N_{G}(L) / C_{G}(L)\right|=|G / L|=2$ and $L$ is an inner subgroup of $G$, we have $|\operatorname{Aut}(L)|=2$ and $|L|=3$. Hence $|G|=6$ and $\tau$ inverts the elements of $L$. If follows $G=S_{3}$.

(2) If $|S| \geqq 4$ and if every abelian normal subgroup of $S$ is cyclic, then $S=Q_{8}$.

Proof. By Lemma 1, we have $|Z(S)|=2$ and $S$ is non-abelian. $S$ has a cyclic subgroup $M$ of index 2 ([2] Satz III. 7.6). The automorphism group of cyclic 2-group is also 2-group. Since $M$ is an inner subgroup of $G$, we have $S / M=$ $\operatorname{Aut}(M)$. This implies $|\operatorname{Aut}(M)|=2,|M|=4$ and $|S|=8$. There is two non-abelian groups of order 8 , that is $Q_{2}$ and the dihedral group $D_{8}$. Assume $S=D_{8}$. Then $S$ contains an elementary abelian subgroup $K$ of order 4 . There exists an automorphism $\tau$ of $K$ of order 2. Since $K$ is an inner subgroup of $G$, we can find $y \in N_{G}(K)$ that induces $\tau$ on $K$. We can take $y$ to be 2-element. Then the group $\langle y\rangle K$ has order 8 , and it is a 2-Sylow subgroup of $G$. Hence $K$ is a non-cyclic abelian normal subgroup of a 2-Sylow subgroup of $G$. This contradicts the assumption.

By way of contradiction, we suppose $G$ is a minimal counter example for Theorem 3. We have $|S| \geqq 4$ by (1) and $S=Q_{8}$ by (2). Let $\tau$ be the unique involution of $S$.

(3) $C_{G}(\tau)$ is an AI-group.

Proof. Take any abelian subgroup of $K$ of $H=C_{G}(\tau)$ and any automorphism $\sigma$ of $K$.

In the case $|K|=$ odd, we extend $\sigma$ to $\sigma^{\prime} \in \operatorname{Aut}(\langle\tau\rangle \times K)$. Since $\langle\tau\rangle \times K$ is an inner subgroup of $G$, we can find $g \in N_{G}(\langle\tau\rangle \times K)$ that induces $\sigma^{\prime}$ on $\langle\tau\rangle \times K$. Then $g \in C_{G}(\tau)=H$ and $g$ induces $\sigma$ on $K$.

In the case $|K|=$ even, we have $\tau \in K$ since $\tau$ is the unique involution of $H$. Since $K$ is an inner subgroup of $G$, we can find $g \in N_{G}(K)$ that induces $\sigma$ on $K$. $\sigma$ fixes $\tau$, and we have $g \in C_{G}(\tau)=H$.

In any case $\sigma$ is induced by an element of $H$. This means $H$ is an $A I$-group.

(4) $C_{G}(\tau)=G$ 
Proof. Assume $C_{G}(\tau)<G$. By (3), $C_{G}(\tau)$ is an $A I$-group. Hence we have $C_{G}(\tau)=S$ by the minimality of $G$. Let $Q$ be a subgroup of $S$. Since $N_{G}(Q) \cong C_{G}(\tau)$ $=S, N_{G}(Q) / C_{G}(Q)$ is a 2-group. Hence we can apply Frobenius' theorem $([1]$, Th. 7.4.5) to $S$, and $S$ has a normal p-complement $K$ in $G$. Since $\tau$ induces a fixed-point-free automorphism of $K, K$ is abelian ([1] Th. 10.1.4). By Lemma 1, a $p$-Sylow subgroup of $K$ has order $p$. Hence $K$ is cyclic and $\operatorname{Aut}(K)$ is abelian. On the other hand, we have $C_{S}(K)=1$ since $\tau \notin C_{S}(K)$. Hence $S=\operatorname{Aut}(K)$. This is a contradiction.

(5) 3 divides $|G|$.

Proof. Let $p$ be the minimal odd prime that divides $|G|$. Let $\langle x\rangle$ be a cyclic subgroup of order $p$. Since $\langle x\rangle$ is an inner subgroup of $G, N_{G}(\langle x\rangle) / C_{G}(\langle x\rangle)$ is a cyclic group of order $p-1$. By the minimality of $p, p-1=2^{m}$ for some integer $m$. But a 2-Sylow subgroup of $N_{G}(\langle x\rangle) / C_{G}(\langle x\rangle)$ is a four group. Hence $m=1$ and $p=3$.

(6) 3-Sylow subgroups of $G$ have order 3 .

Proof. Let $R$ be a 3-Sylow subgroup of $G$. Assume $|R|>3$, then $R$ is nonabelian by Lemma 1 . Hence $R$ contains an elementary abelian subgroup $L$ of order 9. $|\operatorname{Aut}(L)|=|G L(2,3)|=2^{4} \cdot 3$. This contradicts the fact that $L$ is an inner subgroup of $G$ and $S=Q_{8}$.

(7) $|G|>2^{3} \cdot 3$.

Proof. Let $R$ be a 3-Sylow subgroup of $G$. Assume $G=S R$. By Lemma 3, we have $C_{G}(R)=T \times R$ and $T$ is an $A I$-group. By the minimality of $G, T=S_{1}, S_{2}$, $S_{3}$ or $Q_{8}$. From $\tau \in T, T \neq 1$. Since $T$ is $3^{\prime}$-group, $T \neq S_{3}$. If $T=Q_{8}$, then $R \cong Z(G)$. This contradicts the fact that $R$ is an inner subgroup of $G$. Hence $T=\langle\tau\rangle$ and $C_{G}(R)=\langle\tau\rangle \times R$. Since $R$ is an inner subgroup, $\left|N_{G}(R) / C_{G}(R)\right|=|\operatorname{Aut}(R)|=2$, and we have $\left|G: N_{G}(R)\right|=2$. This means the number of 3-Sylow subgroups of $G$ equals to 2, which contradicts Sylow's theorem.

(8) 7 divides $|G|$.

Proof. Let $p$ be the minimal prime that divides $|G|$ and is greater than 3 . Let $\langle x\rangle$ be a cyclic subgroup of order $p$. As in the proof of (5), $p-1=2^{m} 3^{k}$ for some integers $m$ and $k$. Since 2-Sylow subgroups of $N_{G}(\langle x\rangle) / C_{G}(\langle x\rangle)$ are contained in an elementary abelian group of order 4 and 3-Sylow subgroups have order at most 3 , we have order at most 3 , we have $p-1=2 \cdot 3$ and $p=7$.

(9) 7-Sylow subgroups of $G$ have order 7.

Proof. As the proof of (6).

Let $R$ be a 3-Sylow subgroup of $G$ and $P$ be a 7-Sylow subgroup of $G$. By (6) and (8), $|R|=3$ and $|P|=7$. Set $L=N_{G}(P)$. 
(10) $C_{G}(R)=\langle\tau\rangle \times R, \quad C_{G}(P)=\langle\tau\rangle \times P$.

Proof. By Lemma 3, $C_{G}(P)=T \times P, T=S_{1}, S_{2}, S_{3}$ or $Q_{8} . \quad T \neq 1$ since $\tau \in T$. Since $\tau$ is the unique involution, $T \neq S_{3}$. Since there is an 2-element that inverts the elements of $P$, we have $T \neq Q_{8}$. Hence $T=\langle\tau\rangle$ and $C_{G}(P)=\langle\tau\rangle \times P$. The proof of $C_{G}(R)=\langle\tau\rangle \times R$ is as well as above.

(11) $\left|N_{L}(R)\right|=12$.

Proof. $\quad N_{L}(R) / C_{L}(R) \leqq \operatorname{Aut}(R)$ implies $\left|N_{L}(R) / C_{L}(R)\right| \leqq 2$. Here $C_{L}(R)=\langle\tau\rangle \times R$ by (10), hence $\left|N_{L}(R)\right|=6$ or 12 . If $\left|N_{L}(R)\right|=6$, then $\left|L: N_{L}(R)\right|=14$. This means the number of 3 -Sylow subgroups of $L$ equals to 14 , a contradiction.

From (10) and (11), we have $\left|N_{L}(R): C_{L}(R)\right|=2$. There exists a 2-element $x \in N_{L}(R)-C_{L}(R), x$ must be of order 4 . Since $x$ inverts the elements of $R$, we have $\langle x\rangle R /\langle\tau\rangle=S_{3}$. This means that $N_{G}(P) / C_{G}(P)=L /\langle\tau\rangle P$ involves $S_{3}$. This contradicts the fact that $\operatorname{Aut}(P)$ is a cyclic group of order 6 .

Q.E. D.

\section{§. Proof of Theorem 2.}

We shall prove Theorem 2 by way of contradiction, and suppose $G$ is a minimal counter example for Theorem 2. Hence $G$ is a solvable $A I$-group of minimal order that is distinct from $S_{1}, S_{2}, S_{3}$ and $Q_{8}$.

(1) G has no non-trivial normal 2-subgroup.

Proof. Assume there exists a non-trivial normal 2-subgroup $M$ of $G$. Then $\Omega_{1}(Z(M))$ is also a non-trivial normal 2-subgroup of $G$. Hence we may assume $M$ is an elementary abelian 2-group. Since $G$ has one class of involutions by Lemma $2, M^{\#}$ is the set of all involutions of $G$.

We see $|M|=4$. If $|M|=8$, then $G$ involves $G L(3,2)$ since $M$ is an inner subgroup of $G$. But $G L(3,2)$ is non-solvable and contradicts our assumption that $G$ is solvable. If $|M|=2$, then 2-Sylow subgroup of $G$ has only one involution. It is a contradiction by theorem 3 . Hence $|M|=4$.

There exists a 3-element $x$ that induces an automorphism of $M$ of order 3 . Since $|\operatorname{Aut}(\langle x\rangle)|$ is even, $N_{G}(\langle x\rangle)$ has even order. So $N_{G}(\langle x\rangle)$ has an involution and $M$ contains the all involutions of $G$, hence we have $N_{M}(\langle x\rangle) \neq 1$.

Now we consider the group $H=M\langle x\rangle . \quad N_{M}(\langle x\rangle) \neq 1$ implies $\left|H: N_{H}(\langle x\rangle)\right| \leqq 2$. This means the number of 3-Sylow subgroups of $H$ is at most 2. By Sylow's theorem, we get $\langle x\rangle\langle H$. This implies $H=\langle x\rangle \times M$, which contradicts the choice of $x$.

(2) Let $P$ be a p-Sylow subgroup of $G$ for some prome $p$. If $|P|=p$, then $P$ is not normal in $G$.

Proof. Assume $|P|=p$ and $P<G$. From Lemma 3, we have $C_{G}(P)=T \times P$ where $T$ is an $A I$-group. By the minimality of $G, T$ is isomorphic to $S_{1}, S_{2}, S_{3}$ 
or $Q_{8}$. If $T=1$, then $C_{G}(P)=P$. Hence we have $N_{G}(P) / C_{G}(P)=G / P=\operatorname{Aut}(P)$. This implies $G / P$ is cyclic and hence 2-Sylow subgroups of $G$ are cyclic. But this is impossible by Theorem 3. If $T=S_{2}, S_{3}$ or $Q_{8}$, then 2-Sylow subgroup of $C_{G}(P)$ has only one involution. Let $S$ be a 2-Sylow subgroup of $G$, then $S \cap C_{G}(P)$ has only one involution. Since $C_{G}(P)<G$ and $G$ has one class of involutions by Lemma $2, S$ has only one involution, a contradiction by Theorem 3 .

(3) Let $p$ be a prime divisor of $|G|$ greater than 3, then any p-Sylow subgroup of $G$ has order $p$.

Proof. Let $P$ be a $p$-Sylow subgroup of $G$. If $|P|>p$, then $P$ is non-abelian by Lemma 1 , and $P$ contains an elementary abelian subgroup $K$ of order $p^{2}$. This means $G$ involves $\operatorname{Aut}(K)=G L(2, p)$ that is non-solvable, a contradiction.

Now we take a minimal normal subgroup $M(\neq 1)$ of $G$. Since $G$ is solvable, $M$ is an elementary abelian group.

(4) $|M|=9$

Proof. From (1), (2) and (3), $M$ is 3-group and $|M| \geqq 9$. If $|M| \geqq 27$, then $G$ involves $G L(3,3)$ that is not solvable. Hence $|M|=9$.

(5) $G$ is a $\{2,3\}$-group.

Proof. Let $p$ be a prime divisor of $|G|$ greater than 3, and $S$ be a $p$-Sylow subgroup of $G$. From (3), we have $|S|=p$. By Lemma 3, we have $C_{G}(S)=T \times S$ where $T=S_{1}, S_{2}, S_{3}$ or $Q_{8}$. Hence $S$ does not centralize $M$ and $S$ is embedded in $\operatorname{Aut}(M)$. But this is impossible since $|\operatorname{Aut}(M)|=2^{4} \cdot 3$.

(6) $C_{G}(M)$ contains all involutions of $G$.

Proof. If $C_{G}(M)$ has odd order, then a 2-Sylow subgroup of $G$ is isomorphic to a 2-Sylow subgroup of $G L(2,3)$. Hence $|S|=2^{4}$ and $S$ has an element $x$ of order 8. Since $\langle x\rangle$ is an inner subgroup of $G$, we have $N_{G}(\langle x\rangle) / G_{G}(\langle x\rangle)=\operatorname{Aut}(\langle x\rangle)$. This implies that $2^{5}$ divides $|G|$, a contradiction. Hence $C_{G}(M)$ is of even order and contains an involution of $G$. Since $C_{G}(M)<G$, we have (6) by Lemma 2 .

Let $R$ be a 3-Sylow subgroup of $G$. We have $R \supseteq M$. Let $L$ be a maximal abelian normal subgroup of $R$ that contains $M$.

(7) $C_{G}(L)=L$.

Proof. assume $C_{G}(L)>L$. By Lemma $3, C_{G}(L)=T \times L$ where $T$ is a $3^{\prime}$-group and $A I$-group. Hence $T$ must be $S_{2}$ or $Q_{8}$. Let $\tau$ be the involution of $T$ and set $H=C_{G}(\tau)$. Since $\tau$ is the unique involution of $C_{G}(L)$, we have $C_{G}(L) \leqq H$. Since any automorphism of $L$ is induced from an element of $H$, we have $N_{H}(L) / C_{H}(L)$ $=\operatorname{Aut}(L)$. On the other hand, we have $N_{G}(L) / C_{G}(L)=\operatorname{Aut}(L)$ and $C_{G}(L)=C_{H}(L)$. Hence $N_{G}(L)=N_{H}(L)$. Since $G$ has one class of involutions, $\tau$ is contained in the center of some 2-Sylow subgroup of $G$, and hence $H$ contains a 2-Sylow subgroup 
of $G$. $H$ also contains a 3-Sylow subgroup of $G$ since $R \leqq N_{G}(L) \leqq H$. Hence we have $H=G$ from (5), and hence $\tau$ is the unique involution of $G$. This is a contradiction by Theorem 3 .

Now let $x$ be a 2-element that inverts the elements of $L$. By (7), $x$ must be an involution. By (6), $x \in C_{G}(M)$. This is a contradiction since $M \leqq L$.

Q.E.D.

\section{REFERENCES}

[1] Gorenstein, D., “Finite Groups," Harper \& Row, New York, 1968.

[2] Huppert, B., "Endliche Gruppen I," Springer-Verlag, Berlin, 1967.

Department of Mathematics,

Tokyo Institute of Technology. 